\title{
Microporous Organo-Silicates: Functional Hybrid Materials for Room-Temperature Gas Sensing
}

\author{
B. Fabbri' , L. Bonoldi', V. Guidi', G. Cruciani', D. Casotti', C. Malagùi', G. Bellussi ${ }^{2}$, R. Millini', L. \\ Montanari ${ }^{2}$, A. Carati ${ }^{2}$, C. Rizzo ${ }^{2}$, S. Zanardi ${ }^{3}$ \\ 1 University of Ferrara, Department of Physics and Earth Sciences, via G. Saragat 1, 44122, Ferrara, \\ Italy, \\ ${ }^{2}$ Eni Spa, San Donato Milanese Research Center, via F. Maritano 26, 20097, San Donato Milanese, \\ Italy, \\ ${ }^{3}$ Eni S.p.A., Renewable Energy and Environmental Laboratories, via G. Fauser 4, 28100, Novara, \\ Italy \\ barbara.fabbri@unife.it
}

\begin{abstract}
The purpose of this work was to satisfy both materials and technological sciences, on the one hand implementing innovative hybrid materials referred to as ECS (Eni Carbon Silicate) in gas sensors manufacturing, and on the other hand verifying their possible operation at room temperature as a technological progress. The ECS phases were employed as functional materials for the production of films to implement in gas sensing devices. For this research activity the basis of screen printing technology was combined with drop coating technique. In order to create a continuous and homogeneous films, a deep investigation on ECS powders filmability was carried out considering the interaction with different solvents, the possible effect of treatments, e.g. sonication and magnetic stirring, and the interaction with components used for screen-printable pastes.

Room-temperature gas measurements were performed to study their possible sensing and electron transport properties. ECS-14 based-sensor showed outstanding performance in humidity conditions and a complete calibration vs. moisture concentration was obtained. ECS-4 and ECS-13 films showed an electrical activity with a low response to benzene and acetaldehyde, respectively.
\end{abstract}

Key words: organic-inorganic materials, Eni Carbon Silicates, film deposition, gas sensor, room-temperature

\section{Introduction}

Nowadays, in order to reach the expectations of sensors market, the research in material and technological sciences have gained a huge development. Moving towards these requirements, hybrid nanomaterials are a fundamental alternative with respect to well-established materials, such as metals, ceramics or plastics cannot fulfill all technological desires for the various new applications [1]. Moreover, room-temperature operation is an extremely valuable goal in gas sensing research, due to intrinsic safety of sensors working in harsh or industrial environment, and the possibility to reduce power consumption and consequently the size of associated electronics [2]. The techniques for sensing films preparation can be classified into two categories depending either on the method used for material synthesis, i.e. whether physical or chemical, or on the way used for film deposition, that is, thick film or thin film technology [3].

ECSs are organic-inorganic hybrid nanopowders obtained by using different bis-silylated organic precursors as silica source, without surfactant agent and $\mathrm{NaAlO}_{2}$ as the source of aluminum, which plays a relevant role favoring the crystallization of ECS's. The novelty of these materials concerns their structures, crystalline aluminosilicate scaffoldings with long-range 3D order, which distinguish them from the previously reported amorphous and "crystallike" silica-based PMOs (Periodic Mesoporous Organosilicas) [4].

We investigated six different ECS phases (4, 5, 9, $12,13,14)$ as functional materials for gas sensing application, studying the filmability of the powders and exposing the devices produced to several analytes.

\section{Study of films depositions}

The novelty of ECS materials required a highly empirical approach. Preliminary studies demonstrated the necessity of working at room temperature, due to structural damages caused by treatments at temperatures higher than $150{ }^{\circ} \mathrm{C}$. The investigation was divided in two steps and the films quality was evaluated by means of Scanning Electron Microscopy (SEM) and X-Ray Diffraction (XRD) analysis, considering effectiveness of the solvents used for ECS-based solutions, continuity of 
films, adhesion of films to the support, influence of the solvent used on the crystalline structure.

Firstly, ECS powders were mixed with different solvents, as dispersing medium, to investigate the solubility and the filmability of the materials. Several solvents were tested as function of their polarity: water, acetic acid, methanol, ethanol, acetonitrile, acetone, dichloromethane, chloroform, toluene, benzene. Among these solvents, ethanol and acetone showed such effectiveness dispersive/disaggregating properties on the ECS films deposited by drop-coating onto silicon substrates. In particular, ethanol showed the ability to give continuity to the film, whereas acetone revealed a significant disaggregating capacity. The ECS films, deposited starting from suspensions of ethanol or acetone, were not sufficiently compact to obtain repeatable layers for sensing devices. Then in a second step of the research, organic vehicles, generally used in the sensing field for the production of screen-printing pastes based on oxide semiconductors, was used as dispersing medium. Therefore, ECS powders were employed as functional material in the preparation of a screenprintable pastes. The suspensions were treated with two cycles of ultrasounds (each of one hour, $300 \mathrm{~W}$ power at $50{ }^{\circ} \mathrm{C}$ ) and deposited by drop-coating onto alumina substrates with gold electrodes. The pristine paste formulation based on ECS phases did not resulted in uniform and continuous films with the presence of significant agglomerates. Then, it was decided to add ethanol for its dispersive properties and acetone in order to enhance the disaggregation of possible agglomerates. The addition of ethanol gave a greater continuity to the film. Moreover, the addition of acetone as disaggregating agent conferred greater uniformity to the film considerably reducing the quantity and size of the agglomerates [5].

\section{Room-temperature gas measurements}

The sensing devices produced were characterized in a dedicated test chamber with several gaseous compounds (alkanes, aldehyde, nitrogen compounds, ketones, alcohols, and aromatic compounds) and humidity. In particular, the sensing performance of the films was studied in roomtemperature operation mode in order to highlight possible technological advantages for such completely new application of ECS materials.

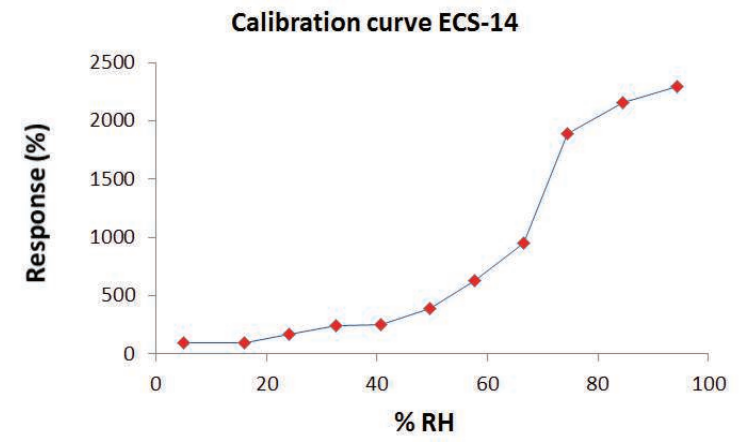

Fig. 1. Responses of ECS-14 based sensor vs. humidity concentration.
ECS-14 based-sensor showed outstanding performance in humidity conditions and a complete calibration vs. moisture concentration was obtained (see Fig. 1) [6]. ECS-4 and ECS-13 films showed an electrical activity with a low response to benzene and acetaldehyde, respectively [5].

\section{Conclusions}

The electrically active films prepared with ECS powders by simple deposition technique can be employed as functional layers in gas sensors. The devices obtained with the ECS hybrids have the properties of speed, reversibility and selectivity fundamental for a good quality electrical response that makes them competitive with respect to systems currently in use. The technological advantage of room-temperature operation and the selectivity are added to the novelty of the application of ECSs as active materials in gas sensing.

\section{References}

[1] G. Kickelbick., Ed. Hybrid Materials: Synthesis, Characterization and Applications; Wiley- $\mathrm{VCH}$ : Weinheim, Germany, 2007; doi:10.1002/9783527610495

[2] B. Fabbri, B. A. Gaiardo,A. Giberti, V.Guidi, C. Malagù, A. Martucci, M. Sturaro, G. Zonta, S. Gherardi, P. Bernardoni, Chemoresistive Properties of Photo-activated Thin and Thick $\mathrm{ZnO}$ Films. Sensors and Actuators B 222, 1251-1256 (2016); doi: 10.1016/j.snb.2015.06.048

[3] V. Guidi, C. Malagu', M.C. Carotta, B. Vendemiati, "Printed films: Materials science and applications in sensors, electronics and photonics" B. in WOODHEAD PUBLISHING SERIES IN ELECTRONIC AND OPTICAL MATERIALS (2012) pp. 278-334

[4] G. Bellussi, A. Carati, E. Di Paola, R. Millini, W.O. Jr Parker, C. Rizzo, S. Zanardi, Crystalline Hybrid Organic-Inorganic Alumino-Silicates. Microporous Mesoporous Materials 113, 252-260 (2008) doi:10.1016/j.micromeso.2007.11.024

[5] B. Fabbri, Eni Carbon Silicates as Crystalline and Mesoporous Hybrids for Gas Sensing. PhD Thesis, University of Ferrara, Italy, (2015)

[6] B. Fabbri, L. Bonoldi, V. Guidi, G. Cruciani, D. Casotti, C. Malagù, G. Bellussi, R. Millini, L. Montanari, A. Carati, C. Rizzo, E. Montanari, S. Zanardi, Crystalline microporous organo-silicates with reversed functionalities of the organic and inorganic components for room.temperature gas sensing, ACS Applied Materials and Inrefaces 9, 24812-24820; doi: 10.1021/ACSAMI.7B02122 